\title{
KIC 10486425: A Kepler eclipsing binary system with a pulsating component
}

\author{
Filiz Kahraman Aliçavuş ${ }^{1,2}$ and Esin Soydugan ${ }^{1,2}$ \\ ${ }^{1}$ Department of Physics, Faculty of Arts and Sciences, Çanakkale Onsekiz Mart University \\ TR-17020 Çanakkale, Turkey \\ ${ }^{2}$ Astrophysics Research Centre and Observatory, Çanakkale Onsekiz Mart University, \\ TR-17020 Çanakkale, Turkey \\ email: filizkahraman01@gmail.com
}

\begin{abstract}
We present frequency analysis of the Kepler light curve of KIC 10486425, an eclipsing binary system with a pulsating component. The parameters of the binary were obtained by modelling the light curve with the Wilson-Devinney program. The residuals from this modelling were subject to Fourier analysis which allowed us to detect 120 periodic terms characteristic for $\gamma$ Dor-type pulsations. The dominant frequency of these changes amounts to $1.3189 \mathrm{~d}^{-1}$.
\end{abstract}

Keywords. stars: binaries: eclipsing, stars: variables: $\gamma$ Dor, stars: individual: KIC 10486425

\section{Introduction}

The Kepler satellite was launched in 2009 in order to discover Earth-like planets around solar-like stars by using transit method (Koch et al. 2010). The mission provided highprecision data for over 200000 stars, contributing remarkably to many branches of astronomy, including asteroseismology. The detached eclipsing binary system KIC 10486425 observed by Kepler was discovered by the TrES project (Devor et al. 2009). In this study, we present light curves and frequency analysis of this star using high-precision Kepler data.

\section{Light curve modelling and frequency analysis}

The parameters of KIC 10486425 were derived by fitting the Kepler light curve by means of the Wilson-Devinney (WD) program (Wilson \& Devinney 1971). For this purpose, we used Kepler Q1 and Q2 data. We adopted Mode 2 which corresponds to the detached configuration. In the analysis, some input parameters were assumed following the Catalog of Eclipsing Binary Stars (Prša et al. 2011). The other parameters were fixed. In particular, bolometric albedos were assumed to be equal to 0.5 (Rucinski 1969) and bolometric gravity-darkening coefficients were set to 0.32 (Lucy 1967). It was also assumed that both components have convective envelopes, that the effective temperature of the primary $T_{1}=7018 \mathrm{~K}$, and that the components of the system rotate synchronously. The other parameters $\left(e, i, T_{2}, \Omega_{1}, \Omega_{2}, q, L_{2}, L_{3}\right)$ were adjusted. We derived two different solutions. In the first solution, a circular orbit was assumed. In the other one, eccentricity and the third-light contribution were chosen as free parameters, starting from 0.08 and 0.013 (Slawson et al. 2011), respectively. The parameters obtained from the light curve analysis are listed Table 1.

Having subtracted the theoretical light curve, we used Kepler Q1 to Q10 data and carried out frequency analysis of residuals with the PERIOD04 program (Lenz \& Breger 2005). We obtained 120 frequencies above the significance limit $(\mathrm{S} / \mathrm{N} \geqslant 4$ ) (Breger 
Table 1. Photometric solutions for KIC 10486425.

\begin{tabular}{|c|c|c|}
\hline Parameter & Solution 1 & Solution 2 \\
\hline$T_{0}$ & $54969.708432^{*}$ & $54969.708432^{*}$ \\
$P($ day $)$ & $5.274816^{*}$ & $5.274816^{*}$ \\
$i\left(^{\circ}\right)$ & $82.314 \pm 0.030$ & $82.549 \pm 0.050$ \\
$T_{1}(\mathrm{~K})$ & $7018^{*}$ & $7018^{*}$ \\
$T_{2}(\mathrm{~K})$ & $5210 \pm 220$ & $5727 \pm 230$ \\
$\Omega_{1}$ & $10.576 \pm 0.006$ & $12.129 \pm 0.027$ \\
$\Omega_{2}$ & $8.096 \pm 0.053$ & $8.055 \pm 0.012$ \\
$\Phi$ & $0.0000 \pm 0.00002$ & $0.0000 \pm 0.00003$ \\
$q$ & $0.4039 \pm 0.0310$ & $0.5907 \pm 0.0120$ \\
$e$ & $0.0^{*}$ & $0.0350 \pm 0.0008$ \\
$L_{3}$ & $0.0^{*}$ & $0.0000 \pm 0.0009$ \\
$L_{1} /\left(L_{1}+L_{2}\right)$ & 0.90 & 0.70 \\
\hline
\end{tabular}

${ }^{*}$ Fixed parameters.
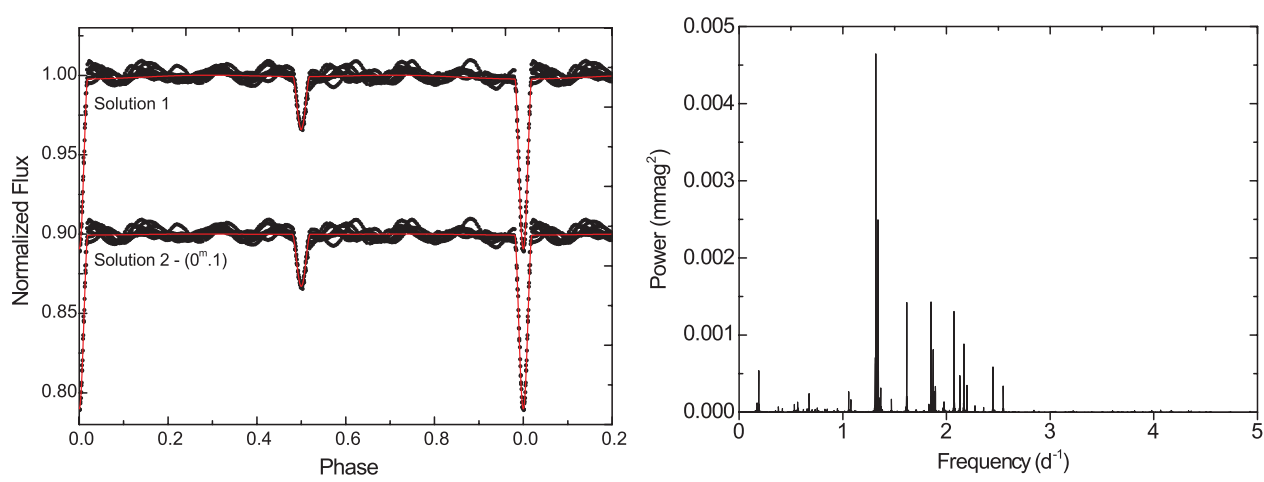

Figure 1. Left: Observed and theoretical (continuous line) light curves. Right: Power spectrum of the residuals from the WD fit.

et al. 1993). Some of these frequencies were harmonics of the system's orbital frequency or combination frequencies. The dominating frequency is equal to $1.3189 \mathrm{~d}^{-1}$; its amplitude amounts to 4.152 mmag. The comparison of theoretical curves with the observational points, and the power spectrum of the residuals from the WD fit are shown in Fig. 1.

\section{Summary and conclusion}

We present the light curve and frequency analysis of KIC 10486425. As a result of this analysis, the parameters of the system were derived and 120 significant periodic terms were found in the residuals. Their frequencies indicate that the primary component of KIC 10486425 is a $\gamma$ Dor-type star with the main pulsation period of about $0.76 \mathrm{~d}$.

\section{References}

Breger, M., Stich, J., Garrido, R., et al. 1993, A\&A, 271, 482

Devor, J., Charbonneau, D., O'Donovan, et al. 2009, AJ, 135, 850

Koch, D. G., Borucki, W. J., Basri, G., et al. 2010, ApJ, 713, L79

Lenz, P. \& Breger, M. 2005, CoAst, 146, 53

Lucy, L. B. 1967, ZfA, 65, 89

Prša, A., Batalha, N., Slawson, R. W., et al. 2011, AJ, 141, 83

Rucinski, S. M. 1969, AcA, 19, 245

Slawson, R. W., Prša, A., Welsh, W. F., et al. 2011, AJ, 142, 160

Wilson, R. E. \& Devinney, R. J. 1971, ApJ, 166, 605 